Meta

Journal des traducteurs

Translators' Journal

\title{
Isosémie et traduction
}

\section{René Poupart}

Volume 39, numéro 1, mars 1994

La traduction et l'interprétation dans la Belgique multilingue

URI : https://id.erudit.org/iderudit/003862ar

DOI : https://doi.org/10.7202/003862ar

Aller au sommaire du numéro

Éditeur(s)

Les Presses de l'Université de Montréal

ISSN

0026-0452 (imprimé)

1492-1421 (numérique)

Découvrir la revue

Citer cet article

Poupart, R. (1994). Isosémie et traduction. Meta, 39(1), 116-121.

https://doi.org/10.7202/003862ar d'utilisation que vous pouvez consulter en ligne.

https://apropos.erudit.org/fr/usagers/politique-dutilisation/ 


\section{ISOSÉMIE ET TRADUCTION}

RENÉ POUPART

Université de Mons-Hainaut, Mons, Belgique

Le problème du sens est inéluctablement au centre de celui de la traduction. Lorsqu'on distinguait le sens et la forme, il était courant de considérer que l'opération traduisante avait pour objectif primordial de restituer le sens. Tout le sens ou, du moins, le maximum de sens. Quant à la forme, c'est-à-dire les sous-codes stylistiques du texte, on ne s'étonnait pas de la résistance qu'ils offraient au traducteur et l'on admettait qu'ils fussent sacrifiés lorsque l'intégrité du sens était en cause.

La linguistique textuelle a remis en question la dualité sens/forme (ou expression/ contenu) et la sémantique, en créant le concept d'isotopie (Greimas 1970; Pottier 1974), a abouti à montrer que le sens lui-même avait une forme. L'isotopie désigne les phénomènes auxquels le texte doit sa cohérence sémantique, fondée sur une continuité thématique (Pottier 1974: 36). L'isosémie (Pottier 1974: 86), communauté de sèmes au sein d'une séquence, dessine les linéaments d'une trame sémantique. Celle-ci, en contribuant à créer la densité du texte, peut être une des composantes de sa spécificité esthétique.

C'est pourquoi le repérage des isotopies est d'une grande importance en traduction. Le respect scrupuleux par le traducteur de la trame isosémique figure parmi les critères permettant d'apprécier la qualité de son travail. En revanche, ne pas discerner la présence d'un système isosémique dans le texte peut être à l'origine d'importantes erreurs.

Dans une perspective pédagogique, on ne saurait trop insister sur l'importance de cette homogénéité sémantique lorsqu'il s'agit d'enseigner à de futurs traducteurs les comportements essentiels en face du texte.

On voudrait produire ici quelques exemples significatifs.

Voici, pour commencer, un texte portugais qui a fait l'objet d'une traduction commentée par un étudiant de maîtrise en traduction portugais-français. Il s'agit d'un article paru dans le Diário de Notícias, quotidien de Lisbonne, le 15 mai 1988 et intitulé : Negritude repensada no Brasil cem anos depois da abolição (La négritude au Brésil, cent ans après l'abolition de l'esclavage):

As raízes e o sangue, o passado remoto e a fermentáço no Novo Mundo têm sido, este ano em que se comemora o Centenário da Aboliçäo da Escravatura, os ingredientes de uma renovação cultural que promete prosseguir, multifacetada, na realidade de todos os brasileiros. É impossivel pensar em Brasil sem recorrer as imagens históricas da escravidäo negra, sem sentir as multiplas misturas de negros, índios e brancos, sem descobrir o tom mulato como símbolo de brasilidade. E é também impossivel percorrer o quotidiano das cidades ou os lugares mais afastados do país continente sem tocar muito de perto uma dimensão de raça embutida nos planos mais profundos da identidade brasileira.

\section{Traduction française :}

Les racines et le sang, le passé lointain et la fermentation dans le Nouveau Monde, tels ont été, en cette année où l'on commémore le centenaire de l'abolition de l'esclavage, les ingrédients d'un renouveau culturel qui promet de se poursuivre dans la réalité de tous les Brésiliens. Il est impossible de penser au Brésil sans avoir recours aux images historiques de l'asservissement des Noirs, sans percevoir les nombreux métissages entre Noirs, Indiens et Blancs, sans découvrir que la tonalité mulâtre est le symbole du peuple brésilien. Il est tout 
aussi impossible de sillonner au quotidien les villes ou les endroits les plus reculés du payscontinent sans toucher de très près à une dimension raciale qui imprègne les structures les plus profondes de l'identité brésilienne!

Deux isosémies traversent parallèlement le premier paragraphe de cet article. Appelons la première l'isosémie historique, représentée par les lexies raízes (1.1), passado (1.1), imagens históricas (1. 5-6).

La seconde est l'isosémie génétique qui exprime les lexies sangue (1.1), fermentação (1.1), ingredientes (1.3), multiplas misturas de negros, indios e brancos (1.6-7).

Nous remarquerons qu'à deux reprises, la conjonction $e$ (et) relie deux termes appartenant aux deux isosémies différentes (raízes e sangue, passado remoto e fermentação). Cette structure parallélistique constitue une marque stylistique qui renforce le système isosémique du texte. suivante :

Rien de tout cela n'avait été remarqué par cet étudiant qui proposait la traduction

Les origines et la race, un lointain passé, l'implantation dans le Nouveau Monde: tels ont été, en cette année où l'on commémore le centenaire de l'abolition de l'esclavage, les éléments d'un renouveau culturel qui...

et justifiait sa traduction de fermentação par implantation au moyen d'une explication extravagante:

Bien que fermentation existe aussi en français, il n'a pas du tout la connotation de son équivalent portugais, qui évoque de manière imagée l'installation des esclaves dans le Nouveau Monde...

Nous avons affaire tout simplement à un contresens, d'autant plus grave qu'il supprime un des éléments de l'isosémie génétique. Si le traducteur avait repéré la présence des relations isotopiques du texte, il aurait compris qu'il fallait choisir la traduction directe fermentation, dont les valeurs sémantiques (transformation d'une substance d'origine organique) participent à l'isosémie génétique. Il aurait, d'autre part, fait urie traduction directe de as razes e o sangue: les racines et le sang. Il a décidé, au contraire, de moduler en supprimant les métaphores sous prétexte que celles-ci sont ambiguës. Cet argument n'est pas valable. En effet, sang est une métaphore lexicalisée en français depuis longtemps («sang se dit de races d'hommes par rapport aux croisements» - Littré, s.v. Sang). Sans doute le terme connote-t-il la langue classique ( Seigneur, je suis Romain et du sang de Pompée», Corneille, Cinna), mais le français courant 1'utilise encore: on parle de sang royal, de sang mêlé. Quant à raizes racines, il mérite aussi d'être conservé, comme le fait, par exemple, le traducteur du roman de l'écrivain américain Alex Haley, Roots (1976): le titre français est Racines.

Ajoutons encore que, si la traducteur avait décelé le système isotopique du texte, il n'aurait pas traduit ingredientes par éléments, car le mot portugais et son correspondant français ingrédients désignent un élément entrant dans la composition d'un mélange (ici de races) et, par conséquent, de cultures.

Le manque d'attention aux isotopies textuelles peut être à l'origine de certaines faiblesses chez des traducteurs chevronnés et de qualité. Voici un exemple provenant de la traduction - excellente au demeurant - qu'a faite Michelle Giudicelli d'un des chefsd'œuvre du romancier portugais Eça de Queiroz, le conte fantastique $O$ Mandarim (Le Mandarin), tout imprégné d'ironie voltairienne.

Le narrateur-héros, Teodoro, évoque une scène se déroulant un dimanche d'été dans la tiédeur ouatée de la paisible pension de famille où il réside: il décrit la patronne, Dona 
Augusta, occupée à frictionner avec du blanc d'œuf les cheveux d'un pensionnaire. Le narrateur termine ainsi l'évocation :

Eu então, enternecido, dizia á deleitosa senhora:

Ai D. Augusta, que anjo que é !

Michelle Guidicelli propose la version française suivante:

Alors, tout attendri, je disais à l'excellente dame:

- Ah! Dona Augusta, vous êtes un ange!

La traduction de deleitosa senhora par excellente dame n'est pas vraiment satisfaisante. C'est une formule stéréotypée qu'un fréquent usage a quelque peu vidée de sa substance. On peut considérer qu'il y a un réel affaiblissement sémantique par rapport a deleitoso qui signifie délicieux, délectable. Cette entropie est d'autant plus regrettable que l'adjectif deleitoso participe au climat de sensualité diffuse que crée un rapport isosémique entre certains faits d'expression du contexte élargi. D'entrée de jeu, la patronne de la pension est appelée a esplêndida Dona Augusta (la splendide Dona Augusta). Le moment où se déroule la scène est qualifié de hora... deliciosa (heure... délicieuse). Évoquant les gestes de la patronne, l'écrivain la décrit arrebitando o dedo minimo branquinho e papudo (levant en l'air son petit doigt blanc et pulpeux); il parle aussi de a fricção mole das carinhosas mãos (la molle friction des mains caressantes).

Ajoutons encore que certains détails, sur le plan des connotations, viennent renforcer cette atmosphère sensuelle: par les fenêtres entrouvertes, pénètrent $o$ bafo da soalheira (le souffle de la canicule) et o arrulhar das rolas (le roucoulement des tourterelles).

Proposons encore un exemple offert par la traduction d'un texte de langue ancienne, le latin, pour montrer comment le repérage des isotopies permet d'affiner l'analyse critique de la démarche traductionnelle.

Il s'agit d'un passage du célèbre texte de Salluste, De Coniuratione Catilinae (La conjuration de Catilina), au début du chapitre XX:

Catilina, ubi eos, quos paulo ante memoraui, conuenisse uidet, tametsi cum singulis multa saepe egerat, tamen in rem fore credens uniuorsos appellare et cohortari, in abditam partem aedium secedit atque ibi, omnibus arbitris procul amotis, orationem huiuscemodi habuit.

On prendra comme référence la traduction la plus récente, celle qui accompagne le texte établi pour la collection dite «Budé» par A. Ernout (Salluste 1967):

Voyant donc réunis ceux que j'ai mentionnés plus haut, Catilina, malgré les nombreux et longs entretiens qu'il avait eus avec chacun d'eux, crut néanmoins utile de leur adresser un appel et une exhortation générale. Il les emmena donc dans une partie retirée de sa maison, et là, tous témoins écartés, il leur tint à peu près ce discours.

Le traducteur fait subir à la longue période d'importantes modifications syntaxiques. Il la scinde notamment en deux parties. Dans la seconde, il substitue au verbe intransitif secedit (inf. secedere) signifiant se retirer, s'en aller à l'écart, le verbe transitif français emmener. Cette modulation maintient le sème mouvement, mais non celui d'éloignement, alors que le verbe latin, en cumulant les deux, participe à la trame isosémique que forment abditam partem (partie retirée), procul amotis (littéralement: loin, éloignés). Sans doute Ernout a-t-il considéré qu'il pouvait sacrifier un élément de la redondance sémantique. Il n'est d'ailleurs pas le seul à agir ainsi ; c'est le cas de trois de ses prédécesseurs: M. Nisard, Ch. Durozoir et F. Richard (Salluste 1850: 39-40; Salluste 1857: 189-191; Salluste 1947: 27-29). 
Pourtant, pareille concentration isosémique, dans un contexte fort restreint n'est pas fortuite, Salluste, dans un texte élaboré autour de la notion de conjuration, a manifestement voulu insister sur le secret dont Catilina veut entourer la réunion des conspirateurs.

La traduction anglaise de J. C. Rolfe (Salluste 1921: 33-39) réussit à restituer la nuance en employant le verbe withdraw:

When Catiline saw before him the men whom I mentioned a short time ago, although he had often had long conferences with them individually, he thought that it would be well to address and encourage the entire body. Accordingly, withdrawing to a private room of the house and excluding all witnesses, he made the following speech.

Il est tout à fait possible de faire de même en français, en modifiant légèrement la version d'Ernout :

Il les entraîne donc à l'écart dans une partie retirée de sa maison, et là, tous témoins éloignés, il leur tint...

Un autre exemple va nous permettre d'apprécier à sa juste mesure le travail d'un grand traducteur, Armel Guerne. Nous le trouvons dans le troisième poème de Die Hymnen an die Nacht de Novalis, dans le passage suivant:

[...] uber der Gegend schwebte mein entbundner, neugeborner Geist

que Guerne traduit ainsi :

[...] sur le paysage flottait mon esprit libéré de ses liens, né à nouveau (Novalis 1975).

On pourrait, à première vue, considérer que la dilution libéré de ses liens est inutile et que libéré suffit en offrant l'avantage de la brièveté. Mais ce serait ignorer le rapport isosémique qui unit entbundner à ce passage du paragraphe précédent :

- da kam aus blauen Fernen - von den Hohen meiner alten Seligkeit ein Dämmerungsschauer - und mit einemmale riß das Band der Geburt - des Lichtes Fessel.

(alors du fond des bleus lointains, de ces hauteurs de ma félicité ancienne, vint un frisson crépusculaire, et par un coup se rompit le lien natal : la chaîne de la lumière) (Novalis 1975).

Un rapport subtil unit les deux séquences, das Band der Geburt (le lien natal) et des Lichtes Fessel (la chaîne de la lumière) à Entbundner, grâce au sème commun attacher. Neugeborner (littéralement: nouvellement né) joue aussi un rôle en favorisant rétrospectivement le rapport associatif de Entbundner avec Entbindung (accouchement). C'est ainsi que la trame isosémique Band der Geburt - entbundner, neugeborner Geist contribue à dynamiser poétiquement le texte qui devient un centre de rayonnement polysémique. C'est pourquoi la dilution de Guerne, libéré de ses liens, restitue, sans doute sous une forme atténuée, ce qui est en filigrane dans le texte allemand. Nous sommes en présence d'une figure de rhétorique que les uns appellent syllepse de sens (Dupriez 1980), les autres antanaclase interne (Morier 1975).

On apprécie mieux encore le travail d'Armel Guerne, quand on le compare à celui de G. Bianquis (Novalis 1949), autre traductrice des Hymnes :

[...] au-dessus du paysage planait mon esprit libéré, régénéré.

Version ferme et serrée, mais qui affaiblit la résonance poétique. 
Alors que l'idéal de la communication ordinaire est d'être univoque pour écarter toute ambiguiité, cette ambiguïté même est, en revanche, cultivée en poésie, parfois systématiquement dans le texte poétique moderne, volontiers polysémantique (Bachelard 1943).

Ce texte poétique moderne déroule souvent plusieurs isotopies simultanées, en organisant conjointement plusieurs systèmes sémantiques qui s'entrecroisent ou s'entrelacent, obligeant le lecteur à des réévaluations rétrospectives. Cette valorisation simultanée de deux systèmes détermine l'effet proprement littéraire du texte, son dynamisme sémantique, sa richesse de résonance poétique. Or, devant pareille poly-isotopie, le traducteur se trouve souvent dépourvu, car la langue-cible ne lui offre pas souvent des polysémies correspondant à celles de la langue-source. Nous avons étudié de ce point de vue le problème que pose la traduction de certains textes des Cantos iberos du poète espagnol Gabriel Celaya (Poupart 1983).

La presse et la publicité modernes recourent fréquemment elles aussi à ces jeux polysémiques et le texte du quotidien portugais Diário de Notícias, cité plus haut, en offre un exemple avec le mot fermentaçáo. Nous avons vu que les rapports isosémiques favorisaient la traduction directe fermentation, au sens de transformation du matériau biologique de la société du Nouveau Monde par le métissage.

Toutefois, au sein du microcontexte, par rapport à passado, on peut comprendre qu'il s'agit aussi de la fermentation sociopolitique en Amérique du Sud. Cette fermentation sociopolitique étant d'ailleurs en rapport avec la structure multiraciale des sociétés sudaméricaines. Ainsi avons-nous affaire de nouveau à une antanaclase interne : le même mot, non répété, cumule deux sens qui, dans l'antanaclase ordinaire, sont représentés par la répétition du terme.

Le plus souvent, les rapports isosémiques s'établissent dans des contextes relativement restreints. Le réseau des relations sémantiques se tisse à l'intérieur d'un paragraphe, d'un chapitre, dans les limites d'une strophe ou de l'ensemble des strophes d'un poème.

Il arrive cependant quelquefois que les isosémies se distribuent au sein d'un réseau aux mailles très distendues, dépassant les limites d'un chapitre ou d'un seul poème.

Voici un exemple offert par Belle du Seigneur d'Albert Cohen (Cohen 1986): Ariane, la belle, porte une appréciation sur la robe qu'elle vient de revêtir:

«une robe voilière», sourit-elle, ravie de cet adjectif inventé. (Chap. LXVI: 578).

Des rappels isosémiques de cette métaphore jalonnent tout le chapitre suivant:

Victorieuse en sa robe voilière (LXVII : 580).

$[\ldots]$

[...] nue sous la robe frémissante, parfois envolée en deux ailes battant au vent de la marche. Deux terrassiers s'arrêtèrent de piocher pour contempler la haute fille aux lèvres entrouvertes qui cinglait vers eux (LXVII : 582).

[...]

Le bruit de sa robe secouée par la marche était le claquement d'un voilier cinglant vers une île extraordinaire, et l'amour était le vent qui gonflait les voiles (LXVII : 585).

Cette métaphore, robe voilière, est vraiment un symbole important. Chaque fois que le romancier évoque, au sens étymologique, l'enthousiasme amoureux qu'exalte la radieuse Ariane, la Belle du Seigneur, la métaphore remonte à la surface du texte. Ainsi réapparaîtelle aux chapitres LXXI (pp. 625-630) et CIII (p: 989). Aussi le traducteur d'Albert Cohen doit-il nécessairement respecter avec la plus grande attention les jalons de cette métaphore filée que l'écrivain a plantés de loin en loin dans son texte. Il est tenu d'être scrupuleux de ce point de vue parce que les anticipations subtiles sont une caractéristique de l'écriture de l'auteur de Belle du Seigneur. En voici une qui concerne le 'même 
chapitre LXVII où Cohen file sa métaphore navale aux connotations baudelairiennes. Au chapitre LXIII, le monologue intérieur du vieil oncle médecin, l'oncle Gri, méditant sur la difficulté de traduire ce vers de l'Enéide de Virgile: Et vera incessu patuit dea (Et sa marche la révèle déesse), puis après un appel téléphonique d'Ariane, jugeant qu'elle ressemblait à la déesse apparue à Enée, la chasseresse aux genoux découverts (LXIII: $562,563,565)$ annonce subtilement la promenade exaltée d'Ariane, au chapitre LXVII, lorsque, nue sous sa robe voilière de toile, sensuellement sûre de sa beauté, elle marche triomphalement, telle une déesse:

\section{une déesse devant elle, dans la glace d'une bijouterie (LXVII : 581) [...] \\ [...] \\ Marche triomphale de la haute nymphe allant à larges enjambées, $[\ldots]$}

Marche triomphale de l'amour, marche d'Ariane, déesse devenue le long des blés sous le vent chaud courbés. Au contour de la route, trois vierges aux tresses de miel apparurent [...]. Mais lorsqu'elles le croisèrent, elles se turent parce qu'elle rayonnait de la majesté du bonheur, se turent et saluèrent la déesse aux ondes d'or bronzé qui sourit et passa (LXVII : 584) [...].

$[\ldots]$

Auguste, elle allait, mue par l'amour comme autrefois ses saurs des temps anciens, [...] allait, immortelle en sa marche,[...] (LXVII : 585).

Attentif à repérer le jeu de pareille corrélation, le traducteur est tenu d'avoir une perception globalisante du texte et en même temps très méticuleuse dans le détail, en somme très philologique, qui fait de lui le peseur de mots dont parlait Valéry Larbaud (Larbaud 1946), en donnant une valeur méliorative à une expression empruntée à Aulu-Gelle.

\section{RÉFÉRENCES}

BACHELARD, Gaston (1943): L'air et les songes, Essai sur l'imagination du mouvement, Paris, José Corti, $286 \mathrm{p}$.

COHEN, Albert (1986): Belle du Seigneur, Paris, NRF, Bibliothèque de la Pléiade.

DUPRIEZ, Bernard (1980) : Gradus. Les procédés littéraires (Dictionnaire), coll. 10/18, $434 \mathrm{p}$.

ECA DE QUEIROZ (1935): O Mandarim, Porto, Livraria Lello, 5.

ECA DE QUEIROZ (1985): Le Mandarin, traduction et notes de Michelle Giudicelli, préface de António Coimbra Martins, Paris, Éditions de la Différence, $76 \mathrm{p}$.

GREIMAS, A. J. (1966) : Sémantique structurale, Paris, Larousse, pp. 53-54 et 69-101.

LARBAUD, Valery (1946) : Sous l' invocation de Saint Jérôme, Paris, N.R.F., 82 p.

MORIER, Henri (1975) : Dictionnaire de poétique et de rhétorique, Paris, PUF, p. 14 s.v. antanaclase.

NOVALIS (1949): Hymnes à la nuit, (Hymnen an die Nacht, Cantiques (Geistliche Lieder), traduction G. Bianquis, Paris, Aubier, Éditions Montaigne, collection bilingue des classiques étrangers, pp. 84-85.

NOVALIS (1975): Euvres complètes. I. Romans Poésies Essais, éd. établie, traduite et présentée par Armel Guerne, Paris, N.R.F., 1975, III, 256 p.

POTTIER, Bernard (1974) : Linguistique générale, théorie et description, Paris, Klincksieck.

POUPART, René (1983) : «Les polysémies en poésie: le problème de leur traduction. Le cas des Canto iberos de Gabriel Celaya», Vingt ans d'enseignement et de recherche en traduction et en interprétation de conférence, Université de Mons-Hainaut, pp. 145-153.

SALLUSTE (1850): De coniuratione catilinae, traduction M. Nisard, Paris, pp. 39-40.

SALLUSTE (1857) : traduction Ch. Durozoir, Paris, Garnier, pp. 189-191.

SALLUSTE (1921) : traduction J. C. Rolfe, Londres, New York, Loeb, pp. 33-39.

SALLUSTE (1947) : traduction F. Richard, Paris, Garnier, pp. $27-29$.

SALLUSTE (1967) : texte établi et traduit par A. Ernout, Paris, Belles Lettres, coll. Budé, pp. 73-76. 